\title{
Complete characterization of attosecond photoelectron wave packets
}

\author{
D. M. Villeneuve $\odot,{ }^{1}$ Peng Peng, ${ }^{1,2}$ and Hiromichi Niikura $\odot^{3, *}$ \\ ${ }^{1}$ Joint Attosecond Science Laboratory, National Research Council and University of Ottawa, \\ 100 Sussex Drive, Ottawa, Ontario, Canada, K1A0R6 \\ ${ }^{2}$ School of Physical Science and Technology, ShanghaiTech University, Shanghai 201210, China \\ ${ }^{3}$ Department of Applied Physics, Waseda University, 3-4-1 Okubo, Shinjyuku, Tokyo 169-8555, Japan
}

(Received 14 August 2021; accepted 1 November 2021; published 23 November 2021)

\begin{abstract}
Development of attosecond laser technology allows us to measure electron dynamics such as photoionization delays between different species or electronic states. In general, the measured photoionization phase is a mixture of the spectral phase of the extreme ultraviolet (XUV) pulse and the atomic phases inherent to the optical transitions. Hence, it is difficult to disentangle these phases independently. Here we separate these phases by using an XUV attosecond pulse train containing both even and odd harmonic orders, generated by an 800 and $400 \mathrm{~nm}$ laser pulse, in the presence of the infrared $800-\mathrm{nm}$ pulse. We measure the photoelectron angular distributions as a function of two independently controlled delays, the XUV-IR and the 800-400 nm delays, with attosecond time resolution. We analyze the photoelectron angular distributions to determine the relative amplitudes and phases of each angular momentum component. Using an in situ technique, we determine the phases of the harmonic orders and thereby completely determine the atomic phases. Using the obtained atomic phases and amplitudes, we reconstruct the real and imaginary parts of the continuum wave functions associated with three individual photoionization pathways.
\end{abstract}

DOI: 10.1103/PhysRevA.104.053526

\section{INTRODUCTION}

An optical transition is characterized by the complex transition probability amplitude $d$. In the dipole approximation and the length gauge, $d$ is represented as the product of the electronic field and the transition dipole moment [1]. The transition dipole moment is given by the integral of the electron position and the electronic wave functions of initial and final states. If several final states are populated simultaneously due to selection rules, the individual transition probability amplitudes contribute on the total amplitude. In general, since photoelectron spectroscopy measures a quantity proportional to the square of the probability amplitude $|d|^{2}$, it is difficult to measure the complex value of $d$ directly. Furthermore, decomposing the phase of $d$ into the phase of the electric field and the phases of the individual transition dipole moments is challenging.

Recent advances in attosecond technology have paved the way to retrieve the phase information of a transition probability amplitude [2-13]. High harmonic generation is the mechanism by which attosecond pulses in the extreme ultraviolet (XUV) region are produced. A single attosecond pulse has a broad spectrum [3], whereas an attosecond pulse train

\footnotetext{
*niikura@waseda.jp

Published by the American Physical Society under the terms of the Creative Commons Attribution 4.0 International license. Further distribution of this work must maintain attribution to the author(s) and the published article's title, journal citation, and DOI.
}

has a spectrum composed of peaks at odd multiples of the driving laser frequency. A method referred to as RABBIT measures kinetic energy spectra or momentum distributions of photoelectrons ionized by the attosecond pulse train in the presence of an IR laser field as a function of the XUV and IR delay [4-8]. The intensity of the photoelectron signal is modulated as the delay varies. The relative phase of the intensity modulation carries information on the following two components. One is the harmonic phase, which comes from the fact that each harmonic in the XUV pulse has a different spectral phase. The other is the atomic phase, which consists of the phase of the transition dipole moments associated with different transition pathways. Using the RABBIT experiments alone and without calculations [4,5] or reference atoms [9], it is difficult to disentangle the harmonic phase from the atomic phase as well as further decomposing the atomic phase into the phases of individual transition moments.

Another approach for attosecond dynamics and pulse measurements utilizes the electron recollision process which is the mechanism underlying attosecond XUV pulse generation [10]. The phase, amplitude, and polarization of the attosecond XUV pulse generated from a sample material contain information about molecular orbital structure, electron and molecular dynamics, and band structure of solid materials [11-13]. In a method referred to as in situ measurement, one generates an XUV pulse which has both even and odd harmonics by focusing a fundamental pulse $(\omega)$ combined with its second harmonic $(2 \omega)$ to a sample gas or a solid material. The high-harmonic spectra are measured as a function of the $\omega-2 \omega$ delay. From the modulation of the even harmonic 


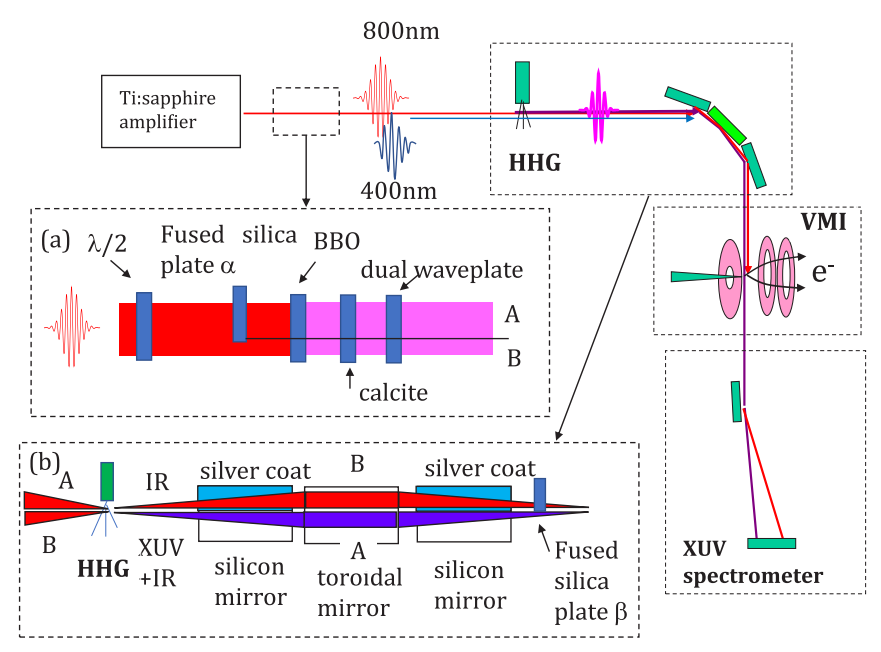

FIG. 1. Experimental setup. The main panel shows the view from top. Side panel (a) shows how to generate the second harmonic and make two delays. The side panel (b) shows how to isolate the XUV pulse from the copropagating IR pulse and adjust the delay between the XUV and IR pulses.

spectral intensity, the spectral phase of the XUV pulse can be retrieved [12].

Here we combine these two attosecond approaches to determine both the harmonic phase and atomic phases of all angular momentum components in a photoelectron wave packet without the need for calculations or reference atoms. We generate attosecond pulse trains which consist of both even and odd harmonic orders by focusing $\omega$ and $2 \omega$ pulses into an argon gas jet [7,14-16]. We ionize neon gas using the XUV pulse in the presence of an IR pulse and record the velocity map images (VMIs) of photoelectrons as a function of two delays, $\omega-2 \omega$ delay $T_{2 \omega}$ and the XUV-IR delay $T_{\mathrm{XUV}}$. Since these two delays are controlled independently, this is a two-dimensional (2D) attosecond experiment. Distinct from a conventional RABBIT experiment which utilizes two-path interference in the photoionization process [4-6], our method employs three-path interference to resolve the phases of partial wave components in the photoelectron wave packets [7]. At each value of $T_{2 \omega}$, we analyze the photoelectron angular distributions measured as a function of $T_{\mathrm{XUV}}$ to obtain the phases and amplitudes of the angular momentum components in three different transition pathways. From the variation of the measured phases with $T_{2 \omega}$, we retrieve how the spectral phase of the even harmonics changes as a function of $T_{2 \omega}$ and find a characteristic $\pi$ phase jump. Next, by measuring the high-harmonic spectra as a function of $T_{2 \omega}$ with the in situ method, we disentangle the atomic phase of each angular momentum component from the harmonic phase. Using the obtained atomic phases and amplitudes, we reconstruct the complex electron wave packets associated with three transition pathways in momentum space.

\section{EXPERIMENTAL SETUP}

Figure 1 schematically shows the experimental setup that was developed and constructed at Waseda University. The experiment is based on an earlier one [7] in which neon was photoionized by both even and odd harmonics in the presence of an IR laser field. A dual-stage, Ti:sapphire laser amplifier (Komodo-Dragon, KMLabs) generates an $1 \mathrm{kHz}, 35 \mathrm{fs}$, 800 -nm laser pulse as a fundamental pulse (IR, $\omega$ ). The estimated IR intensity for generation of high-harmonics is $1.2 \times 10^{14} \mathrm{~W} / \mathrm{cm}^{2}$. The side view (a) shows the optical design before the HHG gas jet. After adjusting the polarization with a $\lambda / 2$ plate, we form two temporally separated beams by inserting a half-moon shaped, 1-mm thickness fused silica plate $\alpha$ to the beam path. The intensity ratio of the upper part, referred to as beam $A$, is larger than that of the lower part, beam $B$. Beam $A$ is used to generate the XUV pulse, and beam $B$ is used as a dressing IR laser pulse. Both beams pass through a $\beta$-BBO crystal to generate the second harmonic ( $\mathrm{SHG}, 2 \omega$ ). The intensity of the $2 \omega$ field is less than $1 \%$ of the $\omega$ field, so that the odd harmonic intensity is not strongly modulated [12]. The calcite plate adjusts the group delay between the $\omega$ and $2 \omega$ pulses so that the two pulses are temporally overlapped at the position where the XUV pulses are generated. The dual waveplate rotates the polarization of the $\omega$ pulse to make it parallel to that of the $2 \omega$. These beams are focused into an argon pulsed gas jet in a vacuum chamber to generate the XUV pulse in which odd and even harmonic orders of the fundamental frequency are produced. Rotating the calcite plate makes a phase delay between the $\omega$ and $2 \omega, T_{2 \omega}$.

The side view (b) shows the optical design in the vacuum chamber schematically. The XUV and IR pulses are reflected off two silicon mirrors. The upper part of the silicon mirror surface is coated by silver while the lower part is uncoated. The intensity of the IR in the beam $A$ are reduced by reflection from the lower part of the silicon mirror set at Brewster angle. Since the SHG intensity is sufficiently low, the residual SHG intensity after the mirrors can be low in spite of the slightly different Brewster angle. Beam $B$ is reflected on the silver coated part of the mirror while almost keeping the intensity. A 1 -mm thickness fused silica plate $\beta$ removes the XUV pulse and temporally isolates the SHG pulse from the IR pulse. The XUV and IR pulses are focused by a 270-mm focal length, gold coated toroidal mirror (ARW Optical Corporation) into an ionization region in the velocity map imaging spectrometer. The fused silica plate $\beta$ adjusts the group delay between the XUV and IR pulses. By rotating the plate to increase the optical path length for the IR pulse relative to the XUV pulse, we make a delay between the XUV and IR pulses $T_{\mathrm{XUV}}$. The IR intensity is estimated to approximately $4 \times 10^{12} \mathrm{~W} / \mathrm{cm}^{2}$. We set the VMI spectrometer so that the electrons are accelerated in the direction perpendicular to the laser propagation direction and the polarization directions of the XUV and IR pulses. The VMI spectrometer is a standard design. The VMI images are recorded on a 2D microchannel plate with a phosphor screen and measured by a CCD camera. We measure the spectra of the XUV pulse by using a flat-field grating (Hitachi) and a microchannel plate.

\section{EXPERIMENTAL RESULTS}

\section{A. Three-path quantum interference in XUV-IR photoionization}

Figure 2(a) illustrates the ionization scheme for neon to produce photoelectron wave packets by three ionization 
(a)

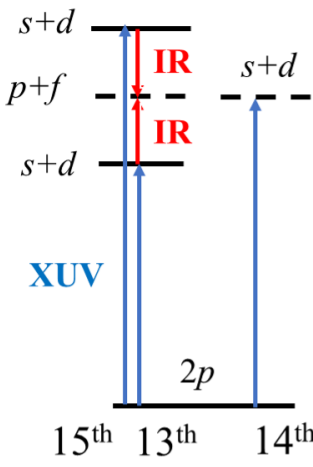

(b)

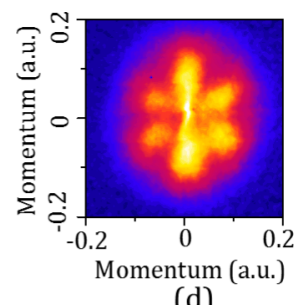

(c)

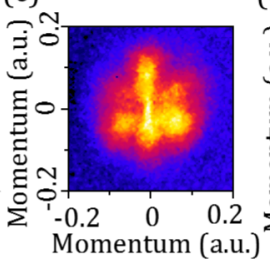

(d)

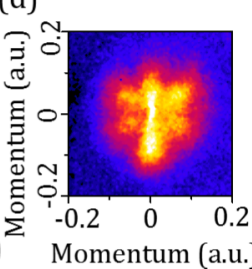

FIG. 2. Three-path quantum interferences. (a) Illustration of an energy diagram for three-path interferences in the photoionization process caused by an XUV and a dressing IR pulse. Two color transitions by harmonic 13 plus one IR photon (H13+IR) and harmonic 15 minus one IR photon (H15-IR) generates a photoelectron wave with $p$ - or $f$ - angular component in the ionization continuum. One-photon ionization by harmonic 14 (H14) generates a $s$ - or $d$ photoelectron wave. By tuning the photon energy of the XUV pulse and the intensity of the dressing IR pulse, we selectively ionize the magnetic quantum number $m=0$. (b) An example of a measured VMI image that is produced by the two-photon ionization processes, H15+IR and H13-IR. (c) and (d) Examples of VMI images when one-photon ionization by $\mathrm{H} 14$ is added to the two-photon ionization process. The images are taken at two different XUV-IR delays ( $\left.T_{\mathrm{XUV}}\right)$ separated by 1.33 fs. Because of the three-path interference, the angular distribution varies with $T_{\mathrm{XUV}}$. The polarization direction of both XUV and IR pulses is indicated by the black arrow.

pathways. An excitation from the $2 p$ ground state of neon with harmonic order H13, through the resonant $3 d$ bound state, followed by one photon absorption of an IR pulse (H13 + IR) generates an electron wave packet with $\ell=1$ ( $p$ wave) or $\ell=3$ ( $f$ wave) in the ionization continuum. Likewise, ionization with $\mathrm{H} 15$ followed by emission of an IR photon (H15 - IR) generates a $p$ wave or $f$ wave. A direct one-photon ionization with $\mathrm{H} 14$ generates a wave packet with $\ell=0(s$ wave) or $\ell=2$ ( $d$ wave). The electron wave packets produced by these three paths have the same kinetic energy, and therefore coherently interfere with each other. We adjusted the IR laser intensity and the XUV photon energy so that photoelectrons with $m=0$ are preferentially produced [17]. In the low kinetic energy region of the VMI images, a characteristic sixfold structure is observed, composed primarily of an $f$ wave $(\ell=3, m=0)$, as shown in Fig. 2(b). The intensity of each lobe in the structure varies with both delays, $T_{2 \omega}$ and $T_{\mathrm{XUV}}$, due to three-path interference. In Figs. 2(c) and 2(d), we present examples of VMI images measured at $T_{2 \omega}=0.58 f s$ (see the next section) and at two different $T_{\mathrm{XUV}}$ separated by $T_{0} / 2=1.33 \mathrm{fs}$, where $T_{0}$ is the optical period of the fundamental pulse. The top lobe is strongest in (c), whereas the bottom lobe is strongest in (d). The pattern moves up and down as a function of $T_{\mathrm{XUV}}$ due to interference between final-state photoelectron angular distributions that have even and odd parity.
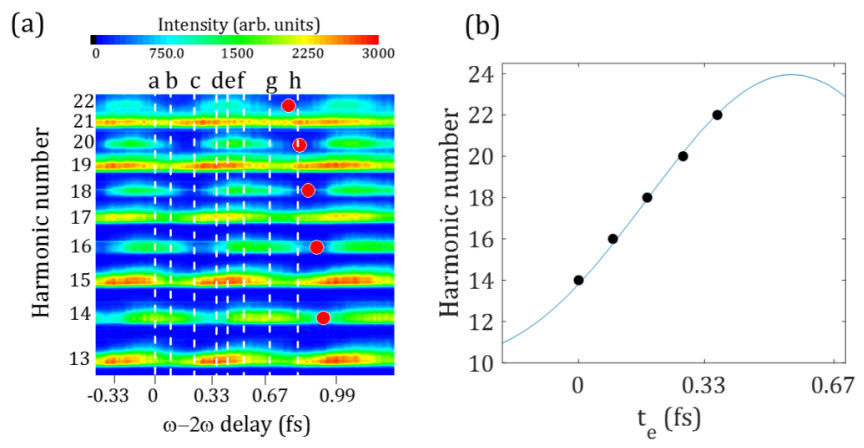

FIG. 3. In situ measurement of the harmonic phase. (a) The highharmonic spectra in argon measured as a function of $T_{2 \omega}$, the delay between the fundamental and second harmonic pulses. The white dotted lines indicate those delays at which VMI measurements were made. The time zero is set at the delay when the H14 intensity is maximized. The red dots mark those times at which the even harmonic intensity minimizes. (b) The emission times (relative to H14) obtained from the measurement (data points). The curve shows the classical calculation of the relative emission time for each harmonic order in argon at the $800 \mathrm{~nm}$ intensity of $1.2 \times 10^{14} \mathrm{~W} / \mathrm{cm}^{2}$.

\section{B. In-situ measurement of the harmonic phase}

Figure 3(a) shows the measured high-harmonic spectra generated in argon as a function of the $\omega-2 \omega$ delay $T_{2 \omega}$. This is the XUV spectrum that photoionizes neon atoms in the VMI. As has been reported elsewhere [12], both odd and even order harmonics are observed. The intensity of all harmonics modulates with a period of $0.66 \mathrm{fs}$. We recover the XUV harmonic phases from these spectra based on the method described in Ref. [12]. We first determine the values of $T_{2 \omega}$ at which the even-order harmonics minimize in intensity and plot them as red dots in Fig. 3(a). We convert the $T_{2 \omega}$ into the emission time, $t_{e}$, relative to that of $\mathrm{H} 14$ using $2 T_{2 \omega}=t_{e}[18,19]$. The curve in Fig. 3(b) represents a classical calculation of the relative emission time for each harmonic order, assuming an $800-\mathrm{nm}$ intensity of $1.2 \times 10^{14} \mathrm{~W} / \mathrm{cm}^{2}$. The calculation is consistent with the experimentally observed emission times $t_{e}$ (data points). We obtain the $t_{e}$ for $\mathrm{H} 13$ and $\mathrm{H} 15$ by inter- and extrapolating the measured emission times with a linear curve. Then the emission time $t_{e}$ of a particular harmonic order $n$ is converted to the harmonic phase by $\phi_{n}^{h}=n \omega_{0} t_{e}$, where $\omega_{0}$ is the angular frequency of the fundamental pulse [5]. The relative harmonic phase is estimated as $\phi_{14}^{h}=0, \phi_{15}^{h}=1.61 \mathrm{rad}$ and $\phi_{13}^{h}=-1.40 \mathrm{rad}$. Harmonic 14 is assigned an emission time and a phase of zero.

\section{Measuring amplitudes and phases of photoelectron angular components}

Next, at a set of $T_{2 \omega}$, we measure the velocity map images as a function of $T_{\mathrm{XUV}}$. Note that in the earlier experiment [7], the measurements were made at a particular $T_{2 \omega}$. The values of $T_{2 \omega}$ we chose in the present experiments are plotted as white dotted lines in Fig. 3(a). We set $T_{2 \omega}=0$ as the time at which the $\mathrm{H} 14$ intensity is maximized. The range of $T_{2 \omega}$ covers one modulation period in the harmonic spectra. At each angle from the vertical polarization axis, we integrate the VMI signal counts along the radial direction in the low kinetic 


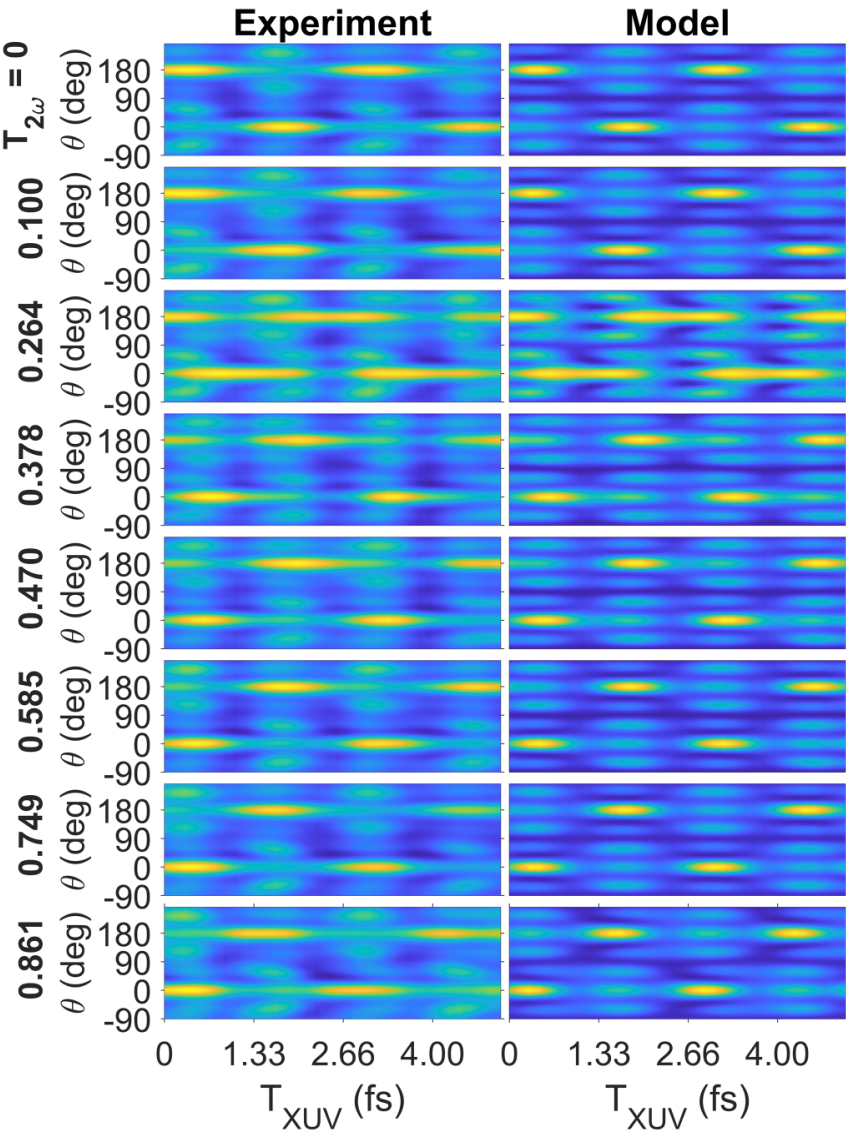

FIG. 4. Photoelectron angular distributions as a function of delays. The experimental (left column) and modeled (right column) photoelectron angular distributions as a function of the XUV-IR delay ( $\left.T_{\mathrm{XUV}}\right)$ at 8 values of the delay between the 800 and $400 \mathrm{~nm}$ driving fields $\left(T_{2 \omega}\right)$. The values of $T_{2 \omega}$ are shown on the left side in $f s$. The derived fitting parameters, amplitudes and phases of angular momentum components, are listed in Table I.

energy region to obtain the photoelectron angular distribution (PAD). We plot the PADs versus $T_{\mathrm{XUV}}$ at eight values of $T_{2 \omega}$ in the left panels of Fig. 4. Here $0^{\circ}$ corresponds to the upper lobe in the VMI images, and $180^{\circ}$ corresponds to the bottom lobe. As has been reported in Ref. [7], the PADs repeat with the full period of the fundamental laser pulse, $2.66 \mathrm{fs}$. It is also apparent from Fig. 4 that the pattern of PADs as a function of $T_{\text {XUV varies with }} T_{2 \omega}$.

Based on the three-path interference model [7], the angular part of the photoelectron wave packet $\left|\psi\left(T_{\mathrm{XUV}}\right)\right|^{2}$ for a fixed $T_{2 \omega}$ is given by

$$
\begin{aligned}
\psi(\theta, & \left.T_{\mathrm{XUV}}\right) \\
= & A_{s} e^{i \phi_{s}^{F}} Y_{00}+A_{d} e^{i \phi_{d}^{F}} Y_{20} \\
& +\left(A_{p 13} e^{i \phi_{p 13}^{F}} Y_{10}+A_{f 13} e^{i \phi_{f 13}^{F}} Y_{30}\right) e^{i \omega_{0} T_{\mathrm{XUV}}} \\
& +\left(A_{p 15} e^{i \phi_{p 15}^{F}} Y_{10}+A_{f 15} e^{i \phi_{f 15}^{F}} Y_{30}\right) e^{-i \omega_{0} T_{\mathrm{Xuv}}}
\end{aligned}
$$

where $A_{s}, A_{d}, A_{p 13}, A_{f 13}, A_{p 15}$, and $A_{f 15}$ represent real amplitudes, $\phi_{s}^{F}, \phi_{d}^{F}, \phi_{p 13}^{F}, \phi_{f 13}^{F}, \phi_{p 15}^{F}$, and $\phi_{f 15}^{F}$ represent phases for each partial wave component, $\omega_{0}$ is the angular frequency of the fundamental pulse, $T_{\mathrm{XUV}}$ is the XUV-IR de-
TABLE I. Fitted amplitudes and phases of partial waves. Amplitude $\left(A_{i}^{F}\right)$ and phase $\left(\phi_{i}^{F}\right)$ values obtained by a global fitting of the experimental VMI data at eight values of $T_{2 \omega}$. The phase of the $s$ wave component is set to zero. All phases are modulo $2 \pi$.

\begin{tabular}{lccccccc}
\hline \hline $\begin{array}{l}T_{2 \omega} \text { delay } \\
\text { (fs) }\end{array}$ & $\begin{array}{c}\text { Ampl. or } \\
\text { phase (rad) }\end{array}$ & $s$ & $d$ & $p 13$ & $p 15$ & $f 13$ & $f 15$ \\
\hline 0.00 & Ampl. & 0.89 & 0.10 & 0.12 & 0.12 & 0.30 & 0.29 \\
& Phase & 0.00 & 1.20 & 1.96 & 0.45 & 5.45 & 3.73 \\
0.100 & Ampl. & 0.87 & 0.12 & 0.12 & 0.13 & 0.31 & 0.32 \\
& Phase & 0.00 & 1.14 & 1.77 & 0.52 & 5.33 & 3.84 \\
0.264 & Ampl. & 0.47 & 0.27 & 0.38 & 0.50 & 0.43 & 0.36 \\
& Phase & 0.00 & 2.61 & 3.27 & 0.71 & 4.60 & 3.60 \\
0.378 & Ampl. & 0.83 & 0.09 & 0.17 & 0.13 & 0.41 & 0.30 \\
& Phase & 0.00 & 0.31 & 5.70 & 3.71 & 2.62 & 0.35 \\
0.470 & Ampl. & 0.87 & 0.07 & 0.13 & 0.13 & 0.33 & 0.29 \\
& Phase & 0.00 & 0.80 & 5.52 & 3.53 & 2.53 & 0.41 \\
0.585 & Ampl. & 0.90 & 0.07 & 0.11 & 0.11 & 0.29 & 0.27 \\
& Phase & 0.00 & 1.17 & 5.24 & 3.62 & 2.36 & 0.55 \\
0.749 & Ampl. & 0.90 & 0.10 & 0.10 & 0.11 & 0.28 & 0.28 \\
& Phase & 0.00 & 5.00 & 2.58 & 1.26 & 5.61 & 4.04 \\
0.861 & Ampl. & 0.90 & 0.07 & 0.07 & 0.15 & 0.26 & 0.31 \\
& Phase & 0.00 & 1.16 & 0 & 3.02 & 2.33 & 0.61 \\
& & & & & & & \\
\hline \hline
\end{tabular}

lay time, $Y_{\mathfrak{l} m}(\theta, \varphi)$ are spherical harmonics, and $\theta$ is the polar angle from the polarization axis of the laser pulses, and $\varphi$ is the azimuthal angle. As shown in Ref. [17], the experiment was able to isolate the magnetic quantum number $m=0$ and hence $Y_{\mathfrak{l} m}$ depends on only $\theta$. Note that the amplitudes and phases are a function of $T_{2 \omega}$.

We use the same analysis as reported in Ref. [7] to determine the phase and amplitude for each partial wave. Independently for each $T_{2 \omega}$, using amplitudes $A_{i}$ and phases $\phi_{i}^{F}$ (where $i$ denotes $s, d, p 13, f 13, p 15$ or $f 15$ ) in Eq. (1) as fitting parameters, we calculate the PADs as a function of $T_{\mathrm{XUV}}$ and compare to the experimentally observed PADs versus $T_{\mathrm{XUV}}$. Using a particle swarm optimization procedure [20], these parameters are globally determined for each value of $T_{2 \omega}$. The optimized fitting parameters are summarized in Table I. Because the global phase cannot be determined, the phase of the $s$ wave $\phi_{s}^{F}$ is set to zero for all $T_{2 \omega}$, and the other phases are relative to that of the $s$ wave. Note that the $s$ and $d$ waves are produced by only the XUV pulse, and so both $\phi_{s}^{F}$ and $\phi_{d}^{F}$ are independent of the delay between the XUV and IR, $T_{\mathrm{XUV}}$; these provide the stationary references for the $p$ - and $f$ -wave components that vary with $T_{\mathrm{XUV}}$. In the right panels of Fig. 4, we plot the calculated PADs as a function of $T_{\mathrm{XUV}}$ using Eq. (1) with the fitted parameters shown in Table I. The calculated patterns of PADs versus $T_{\mathrm{XUV}}$ are consistent with the measured distributions for all $T_{2 \omega}$.

The fitted phases in Table I at $T_{2 \omega}=0.749 f s$ are significantly different from the phases of neighboring data points for all angular momentum components. Since the measured angular distribution pattern observed at $0.749 \mathrm{fs}$ is comparable with those at $0.58 \mathrm{fs}$ (see Fig. 4), the discrepancy might be caused by a failure in the global fitting process. We note that adding $\pi$ to the phases at $0.749 f s$ causes all of the points 
to line up with the other curves. This suggests that the fitting algorithm inverted the phase of the $s$-wave component. For the following analysis, we ignore the data points at $0.749 \mathrm{fs}$.

\section{ANALYSIS}

\section{A. Disentangling the atomic phase from the harmonic phase}

Now we disentangle the atomic phase from the harmonic phase. The atomic phases are relevant to the transition dipole moment and are independent of $T_{2 \omega}$, while the harmonic phases of H13, H14, and H15 depend on $T_{2 \omega}$. The experimentally fitted phases $\phi_{i}^{F}$ shown in Table I can be written as a sum of these two components

$$
\begin{aligned}
\phi_{s}^{F}\left(T_{2 \omega}\right) & =\phi_{14}^{H}\left(T_{2 \omega}\right)+\phi_{s}^{A}, \\
\phi_{d}^{F}\left(T_{2 \omega}\right) & =\phi_{14}^{H}\left(T_{2 \omega}\right)+\phi_{d}^{A}, \\
\phi_{p 13}^{F}\left(T_{2 \omega}\right) & =\phi_{13}^{H}\left(T_{2 \omega}\right)+\phi_{p 13}^{A}, \\
\phi_{f 13}^{F}\left(T_{2 \omega}\right) & =\phi_{13}^{H}\left(T_{2 \omega}\right)+\phi_{f 13}^{A}, \\
\phi_{p 15}^{F}\left(T_{2 \omega}\right) & =\phi_{15}^{H}\left(T_{2 \omega}\right)+\phi_{p 15}^{A}, \\
\phi_{f 15}^{F}\left(T_{2 \omega}\right) & =\phi_{15}^{H}\left(T_{2 \omega}\right)+\phi_{f 15}^{A},
\end{aligned}
$$

where the $\phi_{n}^{H}$ represent the spectral phases of the corresponding harmonic orders, and the $\phi_{i}^{A}$ represent the atomic phases. After substituting the phases defined in Eqs. (2) into Eq. (1) and dividing by $e^{i \phi_{14}^{H}}$, we can relate the phases shown in Table I with the atomic and harmonic phases,

$$
\begin{aligned}
\phi_{s}^{F} & =\phi_{s}^{A}, \\
\phi_{d}^{F} & =\phi_{d}^{A}, \\
\phi_{p 13}^{F}\left(T_{2 \omega}\right) & =\phi_{13}^{H}\left(T_{2 \omega}\right)-\phi_{14}^{H}\left(T_{2 \omega}\right)+\phi_{p 13}^{A}, \\
\phi_{f 13}^{F}\left(T_{2 \omega}\right) & =\phi_{13}^{H}\left(T_{2 \omega}\right)-\phi_{14}^{H}\left(T_{2 \omega}\right)+\phi_{f 13}^{A}, \\
\phi_{p 15}^{F}\left(T_{2 \omega}\right) & =\phi_{15}^{H}\left(T_{2 \omega}\right)-\phi_{14}^{H}\left(T_{2 \omega}\right)+\phi_{p 15}^{A}, \\
\phi_{f 15}^{F}\left(T_{2 \omega}\right) & =\phi_{15}^{H}\left(T_{2 \omega}\right)-\phi_{14}^{H}\left(T_{2 \omega}\right)+\phi_{f 15}^{A} .
\end{aligned}
$$

Since we know the left-hand sides of Eqs. (3) from Table I, we can determine the atomic phases if we know the phase relationship between the harmonics, $\phi_{13}^{H}\left(T_{2 \omega}\right)-\phi_{14}^{H}\left(T_{2 \omega}\right)$ and $\phi_{15}^{H}\left(T_{2 \omega}\right)-\phi_{14}^{H}\left(T_{2 \omega}\right)$.

\section{B. The harmonic phase as a function of $\boldsymbol{T}_{2 \omega}$}

We discuss how the harmonic phases depend on $T_{2 \omega}$. Figure 5(a) plots the experimentally determined phases $\phi_{p 13}^{F}, \phi_{f 13}^{F}, \phi_{p 15}^{F}$, and $\phi_{f 15}^{F}$ as a function of $T_{2 \omega}$ from Table I. Since the intrinsic atomic phase $\phi_{i}^{A}$ is independent of $T_{2 \omega}$, the phase variation is caused only by the harmonic phases. For all four channels, a phase jump is seen at $T_{2 \omega} \sim 0.33 \mathrm{fs}$ when the intensity of $\mathrm{H} 14$ is minimized [see Fig. 3 (a)]. In Fig. 5(b), we plot the phases averaged over the four channels. The averaged phase jump is $3.23 \pm 0.30$ radians, close to $\pi$. In Ref. [14], it was inferred that the even harmonics were $\pi / 2$ out of phase with the neighboring odd harmonics [14], but the variation with the $\omega-2 \omega$ delay was not determined.

The $\pi$ phase jump can be explained by a simple analytical model based on the discussion in Refs. [12,18] and as described in the Appendix. The complex spectral
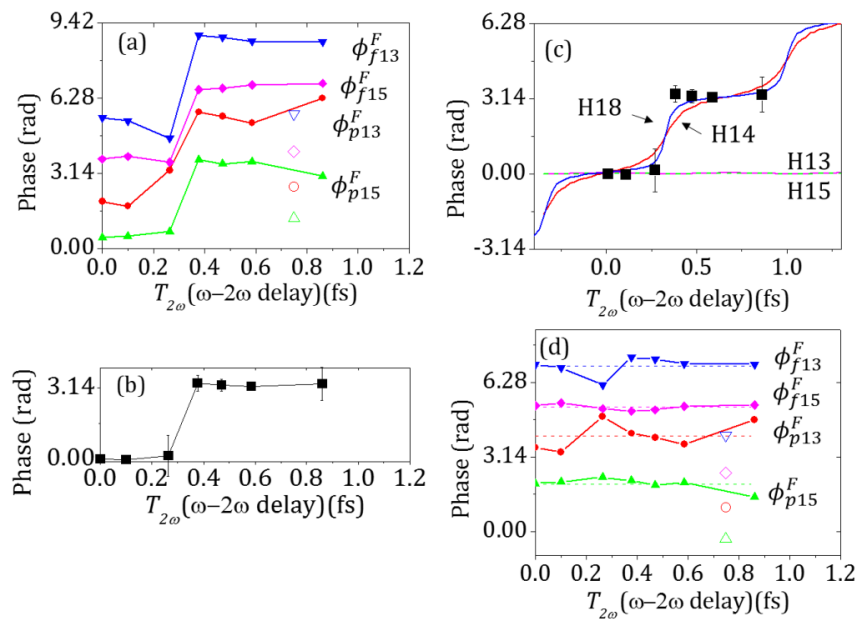

FIG. 5. Phases of photoelectron angular components as a function of $T_{2 \omega}$. (a) The fitted phases $\phi_{p 13}^{F}, \phi_{p 15}^{F}, \phi_{f 13}^{F}$ and $\phi_{f 15}^{F}$ listed in Table I. The data points at $T_{2 \omega}=0.749 \mathrm{fs}$ are significantly shifted from neighboring points due to the fitting procedure (see text). (b) The phases averaged over $\phi_{p 13}^{F}, \phi_{p 15}^{F}, \phi_{f 13}^{F}$ and $\phi_{f 15}^{F}$. The phase at $T_{2 \omega}=0$ is set to zero. The lengths of the error bars are normalized so that the error at $T_{2 \omega}=0$ is zero. A $\pi$ phase jump (3.23 radians) is seen at $T_{2 \omega} \sim 0.33 \mathrm{fs}$. (c) The calculated spectral phases of H14 (red) and H18 (blue) versus $T_{2 \omega}$. The SFA calculation is compared with the experimental data (square data points). The spectral phases for H13 (magenta) and H15 (green) are also plotted. (d) The phases after removing the $\pi$ phase jump. The dashed lines represent the phases averaged over $T_{2 \omega}$ for each partial wave component.

amplitudes of odd and even harmonics are given by $E_{\text {odd }}(\omega)=2 \cos \vartheta\left|E_{0}(\omega)\right| \exp \left(i \phi_{\omega}^{H}\right) \quad$ and $\quad E_{\text {even }}(\omega)=$ $2 i \sin \vartheta\left|E_{0}(\omega)\right| \exp \left(i \phi_{\omega}^{H}\right)=2 \sin \vartheta\left|E_{0}(\omega)\right| \exp \left[i\left(\phi_{\omega}^{H}+\pi / 2\right)\right]$, where $\vartheta$ is the additional phase that is caused by the weak second harmonic field, $E_{0}(\omega)$ is the XUV field amplitude, and $\phi_{\omega}^{H}$ is the spectral phase in the absence of the second harmonic field. The value of $\vartheta$ oscillates near zero as a function of $T_{2 \omega}$ when the second harmonic field is sufficiently weak. At those delays at which $\vartheta=0$, the even harmonic intensity $\left|E_{\text {even }}\right|^{2}$ becomes zero. When $\vartheta$ changes sign, the phase of $E_{\text {even }}$ changes from $\left(\phi_{\omega}^{H}+\pi / 2\right)$ to $\left(\phi_{\omega}^{H}+\pi / 2-\pi\right)=\left(\phi_{\omega}^{H}-\pi / 2\right)$ or vice versa. Hence, the spectral phase of even harmonics changes by $\pm \pi / 2$ at those delays at which the even harmonic intensity is minimized. Thus

$$
\phi_{14}^{H}\left(T_{2 \omega}\right)= \begin{cases}\phi_{14}^{H}+\frac{\pi}{2} & \text { for } T_{2 \omega}<0.33 f s \\ \phi_{14}^{H}-\frac{\pi}{2} & \text { for } T_{2 \omega}>0.33 f s,\end{cases}
$$

where $\phi_{14}^{H}$ is the spectral phase of H14 in the absence of the second harmonic field as determined in Fig. 3.

A calculation based on the strong field approximation (SFA) [21] supports this conclusion. In Fig. 5(c), we plot the relative harmonic phases for $\mathrm{H} 14$ and $\mathrm{H} 18$ as a function of $T_{2 \omega}$. The intensity of the 800 -nm field is $1.2 \times 10^{14} \mathrm{~W} / \mathrm{cm}^{2}$ and $\frac{E_{2 \omega}}{E_{\omega}}=0.02$. Both curves exhibit a stepwise function with a step size of $\pi$ and intersect at the delay when the even harmonic intensity is maximized, $T_{2 \omega}=0.66 \mathrm{fs}$. The calculation confirms the validity of Eq. (4). As expected from the simple model, the odd harmonics H13 (red) and H15 (green) calculated by SFA are almost invariant with $T_{2 \omega}$. 
TABLE II. Atomic phases and amplitudes of the partial wave components. The left side $\left(\phi_{i}^{F}\right)$ shows the averaged fitted phases after removing the $\pm \pi / 2$ phase jump of H14. The right side shows the atomic phases $\left(\phi_{i}^{A}\right)$ and atomic amplitudes $\left(A_{i}^{A}\right)$. The values of $A_{i}^{A}$ are normalized so that the amplitude of $\mathrm{s}$ wave is unity.

Partial Phase Std Dev Partial Atomic Std Dev Partial Atomic wave (rad) (rad) wave phase (rad) (rad) wave amplitude

\begin{tabular}{lccccccc}
\hline$\phi_{s}^{F}$ & 0 & 0 & $\phi_{s}^{A}$ & 0 & 0 & $A_{s}^{A}$ & 1 \\
$\phi_{d}^{F}$ & 1.2 & 0.7 & $\phi_{d}^{A}$ & 1.2 & 0.7 & $A_{d}^{A}$ & 0.10 \\
$\phi_{p 13}^{F}$ & 4.0 & 0.6 & $\phi_{p 13}^{A}$ & -0.86 & 0.6 & $A_{p 13}^{A}$ & 0.12 \\
$\phi_{p 15}^{F}$ & 2.0 & 0.3 & $\phi_{p 15}^{A}$ & 0.39 & 0.3 & $A_{p 15}^{A}$ & 0.12 \\
$\phi_{f 13}^{F}$ & 7.0 & 0.4 & $\phi_{f 13}^{A}$ & 2.08 & 0.4 & $A_{f 13}^{A}$ & 0.31 \\
$\phi_{f 15}^{F}$ & 5.2 & 0.1 & $\phi_{f 15}^{A}$ & 3.63 & 0.1 & $A_{f 15}^{A}$ & 0.29 \\
\hline \hline
\end{tabular}

This is an experimental observation of the $\pm \pi / 2$ (or $\pi$ ) phase jump in the even harmonic emission. With this knowledge, we can remove the harmonic phase dependence on $T_{2 \omega}$. The experimentally determined phases $\phi_{i}^{F}\left(T_{2 \omega}\right)$ include the $\pm \pi / 2$ phase jump through the $\phi_{14}^{H}\left(T_{2 \omega}\right)$ term. We remove the $\pm \pi / 2$ phase jump on both sides of Eqs. (3) using $\phi_{13}^{H}\left(T_{2 \omega}\right)-\phi_{14}^{H}\left(T_{2 \omega}\right)=\phi_{13}^{H}-\phi_{14}^{H} \pm \pi / 2$ and $\phi_{15}^{H}\left(T_{2 \omega}\right)-$ $\phi_{14}^{H}\left(T_{2 \omega}\right)=\phi_{15}^{H}-\phi_{14}^{H} \pm \pi / 2$. We add $\pi / 2$ for $T_{2 \omega}<0.33 f s$ and $-\pi / 2$ for $T_{2 \omega}>0.33 \mathrm{fs}$ to $\phi_{i}^{F}$ and plot the results in Fig. 5(d). The averaged values for $\phi_{i}^{F}$ are plotted as dotted lines and listed in the left panel of Table II.

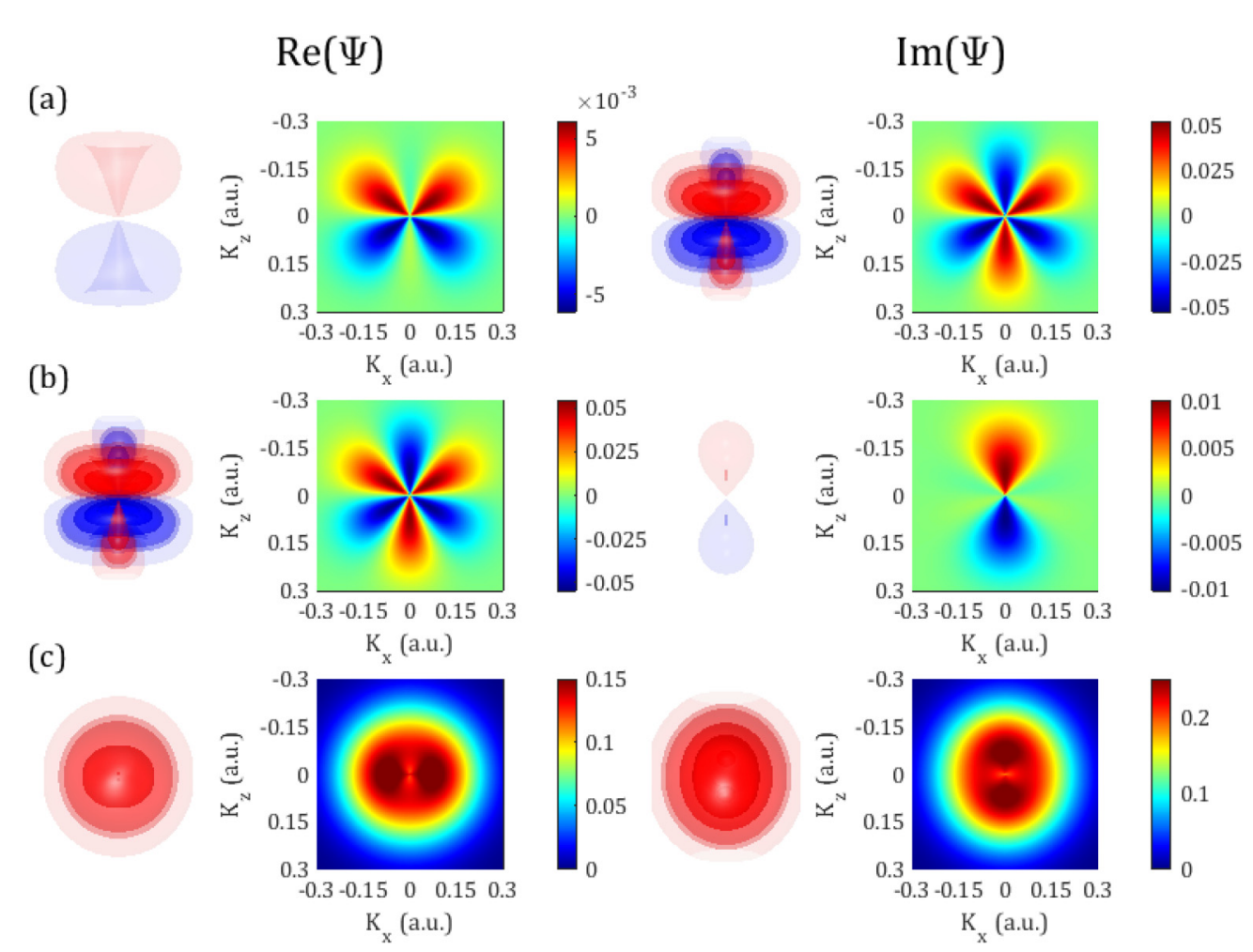

\section{The atomic phases and amplitudes for partial waves}

We now have all the ingredients required to determine the intrinsic atomic phases for each channel. Subtracting the values of $\phi_{15}^{H}-\phi_{14}^{H}=1.61 \mathrm{rad}$ and $\phi_{13}^{H}-\phi_{14}^{H}=-1.40 \mathrm{rad}$ from $\phi_{i}^{F}$ listed in the left panel of Table II, we obtain the atomic phases $\phi_{i}^{A}$ listed in the right panel of Table II. Next, we disentangle the atomic amplitudes $A_{i}^{A}$ from the spectral intensity. At every $T_{2 \omega}$ except $0.26 f s$ when the signal intensity is low, we divide the fitted amplitude listed in Table I by the square root of the measured spectral intensity of each harmonic. The values are averaged over $T_{2 \omega}$ and normalized so that the amplitude of the s wave is unity. The resulting values of $A_{i}^{A}$ are listed in the right panel of Table II as well.

With these atomic phases $\phi_{i}^{A}$ and amplitudes $A_{i}^{A}$, we reconstruct the electron wave functions produced in the ionization continuum by the H13+IR, H14, and H15-IR pathways using the following equations:

$$
\begin{aligned}
& \psi_{H 15-I R}(k, \theta, \varphi) \\
& \quad=\left[A_{p 15}^{A} e^{i \phi_{p 15}^{A}} Y_{10}(\theta, \varphi)+A_{f 15}^{A} e^{i \phi_{f 15}^{A}} Y_{30}(\theta, \varphi)\right] R(k), \\
& \psi_{H 13+I R}(k, \theta, \varphi) \\
& \quad=\left[A_{p 13}^{A} e^{i \phi_{p 13}^{A}} Y_{10}(\theta, \varphi)+A_{f 13}^{A} e^{i \phi_{f 13}^{A}} Y_{30}(\theta, \varphi)\right] R(k), \\
& \psi_{H 14}(k, \theta, \varphi)=\left[A_{s}^{A} e^{i \phi_{s}^{A}} Y_{00}(\theta, \varphi)+A_{d}^{A} e^{i \phi_{d}^{F}} Y_{20}(\theta, \varphi)\right] R(k),
\end{aligned}
$$

where $R(k)$ is the radial wave function. We assume $R(k)$ to be shared for all wave functions. The $R(k)$ is obtained by averaging the observed VMI images over one period of the

FIG. 6. Reconstruction of the photoelectron wave packets. The 3D and 2D re-constructed momentum-space electron wave-packet images produced by photoionization pathways of (a) H15-IR, (b) H13+IR, and (c) H14. The left panels show the real part and the right panels show the imaginary part of the wave packet. The $2 \mathrm{D}$ images are obtained by cutting the $3 \mathrm{D}$ images along the polarization axis, vertical in the figure. We use the atomic phases and amplitudes for each partial wave given in the right side of Table II. 
$T_{\mathrm{XUV}}$ at $T_{2 \omega}=0.58 \mathrm{fs}$, followed by integration of the averaged VMI image over angle and taking a square root of it. We fit the curve with a double Gaussian distribution and remove the very low kinetic energy region which potentially includes the background signals. Figure 6 shows the 3D re-constructed wave-packet images for the ionization pathways of H15-IR, H13+IR, and H14. The 2D images cut along the polarization axis of the 3D images are also shown. The images clearly show that the photoelectron wave packet generated by H15-IR differs from that generated by H13+IR. This is due to the fact that the atomic phase dependence on the photon energy for the $p$ wave is different from that of the $f$ wave. We discuss this in the next section.

\section{CALCULATIONS OF ATOMIC PHASES}

So far, we have obtained the atomic phases for the photoelectron angular momentum components for three ionization pathways. In principle, the result allows us to make a comparison with the calculation of the dipole moment for the transition from the ground state to the particular angular momentum state. We discuss some aspects of the interpretation of the results, although a detailed theoretical modeling is outside the scope of this experimental paper. As shown in Fig. 2(a), there are three pathways that yield the same electron kinetic energy: H14 directly to $s$ and $d$ waves; H15-IR; and H13+IR. The latter channel includes a complex mixing of resonant states around the $3 d$ bound state of neon [17]. The first two channels are transitions from the initial $2 p$ state directly to the continuum. Their phases therefore include the phase of the continuum states, which are scattering states of the ion, including both short-range and long-range (Coulomb) components. To calculate the continuum wave functions, we use a one-electron effective potential for neon [22],

$$
V(r)=-\frac{Z_{c}+a_{1} e^{-a_{2} r}+a_{3} r e^{-a_{4} r}+a_{5} e^{-a_{6} r}}{r},
$$

with $Z_{c}=1, a_{i}=[8.069,2.148,-3.570,1.986,0.931$, $0.602]$. Since the potential is spherically symmetric, the electronic wave function can be represented as a product of a radial and an angular part,

$$
\psi(r, \theta, \varphi)=\sum_{l m} \frac{1}{r} \phi_{l}(r) Y_{l m}(\theta, \varphi) .
$$

Here $\phi_{l}(r)$ is the reduced radial wave function which satisfies the reduced Schrödinger equation with effective potential

$$
V_{l}(r)=\frac{l(l+1)}{2 r^{2}}+V(r) .
$$

The solutions $\phi_{l}(r)$ are determined by integrating the reduced Schrödinger equation using the Numerov algorithm [23]. The wave function is normalized at asymptotically large distances from the core as described in Ref. [24]. The radial solutions determine a scattering phase shift $\eta_{l}=\sigma_{l}+\delta_{l}$, where one of the phases, $\sigma_{l}$, is due to the Coulomb potential, and the other, $\delta_{l}$, is due to the short-range potential specific to neon. These phase shifts are a function of the asymptotic kinetic energy of the electron.

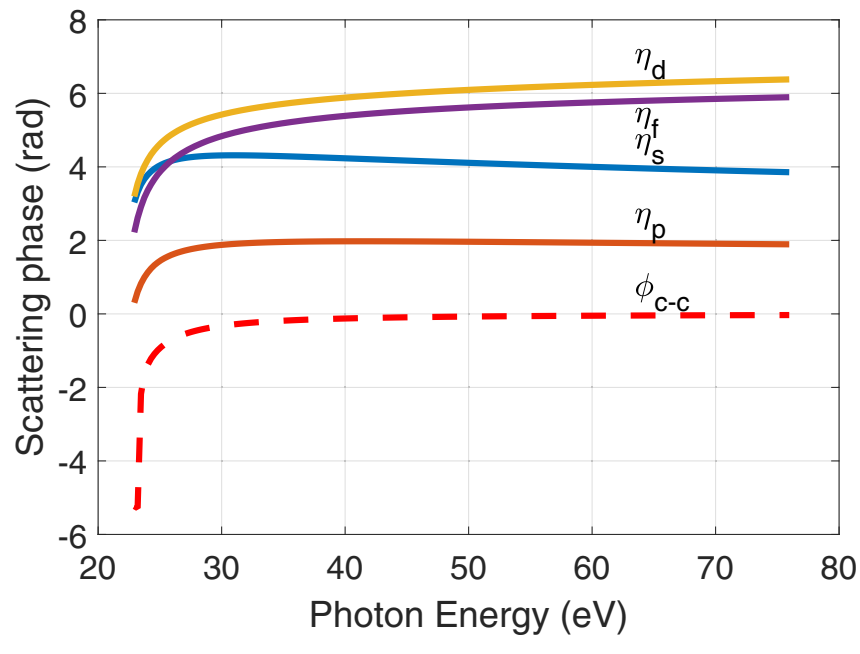

FIG. 7. Scattering phase and continuum-continuum phase. Scattering phases $\eta_{l}$ of the continuum electron wave function for each partial wave component, calculated for neon, as a function of the photon energy. The first ionization potential of neon is $21.56 \mathrm{eV}$. Additional phase shift $\phi_{c c}$ of the photoelectron wave function due to continuum-continuum coupling by the infrared field. The phase diverges quickly as the electron kinetic energy becomes small.

In Fig. 7 we plot the scattering phase shifts corresponding to photoionization of neon as a function of the photon energy. These phase shifts of the continuum electron are involved in all three photoionization channels. For the H14 channel, they are the only phase. For the H15-IR channel, there is an additional phase shift due to continuum-continuum coupling caused by the infrared field. This coupling results in the RABBIT sideband at H15-IR, and also sidebands of higher harmonic orders. This coupling is described in detail by Dahlstrom et al. [25], and we use their notation below. Dahlstrom's Eq. (97) shows the phase of the H15-IR channel. Note that we have experimentally determined the electromagnetic phases $\phi_{\Omega}$ and $\phi_{\omega}$; the phase of the spherical harmonic is zero because $m=0$. In addition to the scattering phase $\eta_{l}$, there is the continuum-continuum phase

$$
\phi_{c c}(k, \kappa)=\arg \left\{\frac{(2 \kappa)^{i Z / \kappa}}{(2 k)^{i Z / k}} \frac{\Gamma\left[2+i Z\left(\frac{1}{\kappa}-\frac{1}{k}\right)\right]}{(\kappa-k)^{i Z\left(\frac{1}{\kappa}-\frac{1}{k}\right)}}\right\} .
$$

Here $k$ is the electron momentum of the single-photon XUV photoionization with $\mathrm{H} 15$, and $\kappa$ is the electron momentum corresponding to the sideband H15-IR. We plot the phase $\phi_{c c}$ as a function of the electron kinetic energy of the H15 electron in Fig. 7. This is similar to Dahlstrom's Fig. 15 (blue curve) except that we show lower kinetic energies.

Both of the phases, $\eta_{l}$ and $\phi_{c c}$ shown in Fig. 7, diverge strongly for small electron kinetic energies. Since the electrons that are detected experimentally by the VMI have kinetic energy of the order of $0.5 \mathrm{eV}$, their phases will be strongly affected. This divergent behavior when the electron kinetic energy approaches zero means that an accurate calculation will be challenging. 


\section{CONCLUSION}

We have measured the photoelectron angular distributions using a velocity map imaging spectrometer as a function of the two independently controlled delays, the XUV-IR delay $T_{\mathrm{XUV}}$ and the $\omega-2 \omega$ delay $T_{2 \omega}$. At each $T_{2 \omega}$, we determined the amplitude and phase of each angular momentum component. Since we employed the three-path interferences using the XUV pulse containing both odd and even harmonics and an IR pulse, the decomposition to each angular momentum component was possible. We observed a clear $\pi$ phase jump of the even harmonics relative to the odd harmonics at the $\omega-2 \omega$ delay when the even harmonic intensity is minimized. Although this phase jump is to be expected from symmetry considerations, the actual shape of the transition has not been previously measured. Then we determined the harmonic phases using the in situ method from the high-harmonic spectra measured as a function of $\omega-2 \omega$ delay. We did not need to use reference atoms or calculations of atomic phases to determine the harmonic phases. Using these two methods together, we fully determined the atomic phases and amplitudes for each angular momentum component.

RABBIT or photoelectron streaking experiments are often used to study attosecond photoionization delays. We connect the RABBIT-type experiments with wave-function imaging. Our method can be a general way to retrieve the atomic phase and amplitude for each partial wave component. First, we isolate the $m=0$ from $m= \pm 1$, otherwise the different $m$ components are incoherently overlapped. Next, we use threepath interference. Because one pathway is created by singlephoton ionization, and the others are created by two-photon ionization, the symmetry of the angular components is different. This allows us to decompose the coherently overlapped $\ell$ states using the angular distribution as a function of the XUVIR delay. Finally, we disentangle the photoionization phase into atomic phase and harmonic phase. Once all phases and amplitudes of the partial waves are obtained, then we can reconstruct the continuum wave functions in momentum space.

These experimental results should encourage detailed theoretical modeling. These calculations will be very challenging, because (1) the low kinetic energy of the electrons leads to very sensitive phase shifts, and (2) one of the channels through a resonant $3 d$ state has significant coupling to other states. These experiments give the clearest measurement to date of photoionization phase shifts for electron wave packets with very low kinetic energy, and will provide a benchmark for detailed calculations.

\section{ACKNOWLEDGMENTS}

H.N. acknowledges useful discussions with S. Kamiya. H.N. acknowledges funding from the Japan Society for the
Promotion of Science (JSPS) Grant-in-Aid for Scientific Research (KAKENHI) Grant No. 18H03903. This paper is a part of the outcome of research performed under a Waseda University Grant for Special Research Projects (Project No. 2021N-005). We acknowledge funding from the Joint Centre for Extreme Photonics (JCEP).

H.N. conducted the experiments and analysis; D.M.V. did data fitting and calculations; H.N., D.M.V., and P.P. discussed the data. All contributed to the writing of the manuscript.

\section{APPENDIX: ANALYTICAL MODEL FOR HIGH-HARMONICS GENERATED BY TWO-COLOR FIELDS}

In this Appendix, we describe an analytical model for the high-harmonic spectra generated by the $800 \mathrm{~nm}(\omega)$ and $400 \mathrm{~nm}(2 \omega)$ based on the discussion by Dudovich et al. [12] and Dahlström et al. [18]. Consider the emitted electric field of a single attosecond pulse created during a halfcycle of the 800-nm field, $e(t)$, and its Fourier transform, $E_{0}(\omega)=\left|E_{0}(\omega)\right| e^{i \phi^{H}(\omega)}$. The spectral phase $\phi^{H}(\omega)$ describes the relative phase between different spectral components. We then add a replica of this single attosecond pulse, produced during the subsequent half-cycle of the $800-\mathrm{nm}$ field, $e(t)=e_{0}(t) e^{i \vartheta}+e_{0}\left(t-\frac{T_{0}}{2}\right) e^{-i \pi} e^{-i \vartheta}$. The second pulse is shifted in time by $T_{0} / 2$, half of the period of the $800-\mathrm{nm}$ field. In addition, by symmetry the second pulse will have the opposite sign of the first pulse, described by the factor $e^{-i \pi}=-1$. We also include an additional phase shift $\vartheta\left(T_{2 \omega}\right)$ describing the perturbation caused by the $400-\mathrm{nm}$ field. This phase shift acts in opposite directions on the two attosecond pulses.

The Fourier transform of $e(t)$ is $E(\omega)=E_{0}(\omega)$ $\left[e^{i \vartheta}+e^{-i \vartheta} e^{-i \pi\left(\frac{\omega}{\omega_{0}}+1\right)}\right]$. The final factor comes from the timeshifting property of the Fourier transform and the relationship $\frac{T_{0}}{2}=\pi / \omega_{0}$. For odd harmonics, $\frac{\omega}{\omega_{0}}=2 m+1$, and for even harmonics, $\frac{\omega}{\omega_{0}}=2 m$, and we have separate expressions, $E_{\text {odd }}(\omega)=2 \cos \vartheta E_{0}(\omega), \quad E_{\text {even }}(\omega)=2 i \sin \vartheta \quad E_{0}(\omega) . \quad$ The spectral intensity of the harmonic orders is the square of these expressions. If there is no 400-nm field, then $\vartheta=0$ and there are no even harmonic orders, as expected. The 400-nm field produces a small phase shift $\vartheta\left(T_{2 \omega}\right)$ that unbalances the interferometer that cancels the even harmonic orders. The even harmonics have an additional $\pm \pi / 2$ phase shift compared to the odd harmonics due to the imaginary prefactor. A phase jump in the even harmonics occurs when $\sin \vartheta$ changes sign, corresponding to the $T_{2 \omega}$ at which the intensity of the even harmonics is zero for a particular harmonic order. From the periodicity of the 800- and 400-nm fields, there will be a phase jump every $T_{0} / 4$ in $T_{2 \omega}$.
[1] P. Atkins and R. Friedman, Molecular Quantum Mechanics, 5th ed. (Oxford University Press, Oxford, 2011).

[2] M. Hentschel, R. Kienberger, Ch. Spielmann, G. A. Reider, N. Milosevic, T. Brabec, P. Corkum, U. Heinzmann, M. Drescher, and F. Krausz, Nature (London) 414, 509 (2001).
[3] T. Popmintchev, M.-C. Chen, D. Popmintchev, P. Arpin, S. Brown, S. Ališauskas, G. Andriukaitis, T. Balčiunas, O. D. Mücke, A. Pugzlys, A. Baltuška, B. Shim, S. E. Schrauth, A. Gaeta, C. Hernández-García, L. Plaja, A. Becker, A. JaronBecker, M. M. Murnane, and H. C. Kapteyn, Science 336, 1287 (2012). 
[4] P. M. Paul, E. S. Toma, P. Breger, G. Mullot, F. Augé, Ph. Balcou, H. G. Muller, and P. Agostini, Science 292, 1689 (2001).

[5] Y. Mairesse, A. de Bohan, L. J. Frasinski, H. Merdji, L. C. Dinu, P. Monchicour, P. Breger, M. Kovačev, R. Taïeb, B. Carré, H. G. Muller, P. Agostini, and P. Salières, Science 302, 1540 (2003).

[6] M. Schultze, M. Fieß, N. Karpowicz, J. Gagnon, M. Korbman, M. Hofstetter, S. Neppl, A. L. Cavalieri, Y. Komninos, Th. Mercouris, C. A. Nicolaides, R. Pazourek, S. Nagele, J. Feist, J. Burgdörfer, A. M. Azzeer, R. Ernstorfer, R. Kienberger, U. Kleineberg, E. Goulielmakis, F. Krausz, and V. S. Yakovlev, Science 328, 1658 (2010).

[7] D. M. Villeneuve, P. Hockett, M. J. J. Vrakking, and H. Niikura, Science 356, 1150 (2017).

[8] C. Cirelli, C. Marante, S. Heuser, C. L. M. Petersson, Á. J. Galán, L. Argenti, S. Zhong, D. Busto, M. Isinger, S. Nandi, S. Maclot, L. Rading, P. Johnsson, M. Gisselbrecht, M. Lucchini, L. Gallmann, J. M. Dahlström, E. Lindroth, A. L'Huillier, F. Martín, and U. Keller, Nat. Commun. 9, 955 (2018).

[9] M. Ossiander, J. Riemensberger, S. Neppl, M. Mittermair, M. Schäffer, A. Duensing, M. S. Wagner, R. Heider, M. Wurzer, M. Gerl, M. Schnitzenbaumer, J. V. Barth, F. Libisch, C. Lemell, J. Burgdörfer, P. Feulner, and R. Kienberger, Nature (London) 561, 374 (2018).

[10] H. Niikura, F. Légaré, R. Hasbani, A. D. Bandrauk, M. Yu. Ivanov, D. M. Villeneuve, and P. B. Corkum, Nature (London) 417, 917 (2002).

[11] J. Itatani, J. Levesque, D. Zeidler, H. Niikura, H. Pépin, J. C. Kieffer, P. B. Corkum, and D. M. Villeneuve, Nature (London) 432, 867 (2004).

[12] N. Dudovich, O. Smirnova, J. Levesque, Y. Mairesse, M. Yu. Ivanov, D. M. Villeneuve, and P. B. Corkum, Nat. Phys. 2, 781 (2006).
[13] G. Vampa, T. J. Hammond, N. Thiré, B. E. Schmidt, F. Légaré, C. R. McDonald, T. Brabec, and P. B. Corkum, Nature (London) 522, 462 (2015).

[14] G. Laurent, W. Cao, H. Li, Z. Wang, I. Ben-Itzhak, and C. L. Cocke, Phys. Rev. Lett 109, 083001 (2012).

[15] C. Chen, C. Hernández-García, Z. Tao, W. You, Y. Zhang, D. Zusin, C. Gentry, P. Tengdin, A. Becker, A. JaronBecker, H. Kapteyn, and M. Murnane, Opt. Express 25, 28684 (2017).

[16] B. Unzicker, J. Vaughan, S. Burrows, B. Tatum, D. Arthur, T. Olsson, S. Jain, T. Hart, P. Stringer, and G. M. Laurent, New J. Phys. 23, 013019 (2021).

[17] P. Patchkovskii, M. J. J. Vrakking, D. M. Villeneuve, and H. Niikura, J. Phys. B 53, 134002 (2020).

[18] J. M. Dahlström, A. L'Huillier, and J. Mauritsson, J. Phys. B 44, 095602 (2011).

[19] J. M. Dahlström, T. Fordell, E. Mansten, T. Ruchon, M. Swoboda, K. Klünder, M. Gisselbrecht, A. L'Huillier, and J. Mauritsson, Phys. Rev. A 80, 033836 (2009).

[20] J. Kennedy and R. Eberhart, Particle swarm optimization, in Proceedings of the International Conference on Neural Networks (ICNN'95) (IEEE, New York, USA, 1995), Vol. 4, pp. 1942-1948.

[21] M. Lewenstein, Ph. Balcou, M. Yu. Ivanov, Anne L'Huillier, and P. B. Corkum, Phys. Rev. A 49, 2117 (1994).

[22] X. M. Tong and C. D. Lin, J. Phys. B 38, 2593 (2005).

[23] D. J. Tannor, Introduction to Quantum Mechanics: A Time Dependent Perspective (University Science Books, Sausalito, CA, 2007).

[24] A. F. Starace, Theory of atomic photoionization, in Handbuch der Physik (Springer, Berlin, 1982), Vol. 31, p. 1.

[25] J. M. Dahlström, A. L'Huillier, and A. Maquet, J. Phys. B 45, 183001 (2012). 